\author{
Available online at www.sciencedirect.com \\ $\because$ ScienceDirect \\ Journal of Hydrodynamics \\ 2010, 22(5), supplement: 832-837 \\ DOI: $10.1016 / \mathrm{S} 1001-6058(10) 60038-8$
}

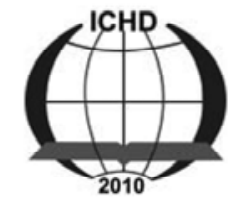

www.sciencedirect.com/ science/journal/10016058

\title{
Numerical study on oil and water two-phase flow in a cylindrical cyclone
}

\author{
Hai-fei Liu, Jing-yu Xu, Ying-xiang $\mathrm{Wu}^{*}$, Zhi-chu Zheng \\ LHO, Institute of Mechanics, Chinese Academy of Sciences \\ Beijing, China \\ *E-mail: yxwu@imech.ac.cn
}

\begin{abstract}
Although traditional hydrocyclones are widely used in industries, there are few studies on cylindrical hydrocyclone. In this work, Euler-Euler multiphase approach and the Reynolds stress turbulence model (RSM) are applied to simulate the flow field characteristic of cylindrical cyclone. The result shows that a low pressure zone is formed in the inner part of cyclone and causes an upward flow. Velocity components distribution, tangential velocity, axial velocity and radial velocity have been calculated to study the effect of oil-water separation efficiency of cylindrical cyclone under the conditions of different flow split-ratio and non-dimensional separation acceleration. These results are helpful to design a separator system in the oil production.
\end{abstract}

KEY WORDS: cylindrical cyclone; oil-water flow; split-ratio; separation efficiency.

\section{INTRODUCTION}

Oil-water separation technology in the petroleum industry becomes more and more serious, especially as the oil fields become more mature and produce more water along with the hydrocarbons. However, the mainly multiphase separators are still based on those conventional vessel-type separators which are bulky, heavy, expensive and long time to separate ${ }^{[1]}$. Recently, the petroleum industry has shown keen interest in compact separators. The Liquid-Liquid Cylindrical Cyclones (LLCC) utilize centrifugal separation technology and have gained extensive attention due to its advantages, including compact geometry, low-weight, low-cost and easy maintenance, etc. The LLCC mainly consists of a vertical pipe with a horizontal tangential inlet and two outlets, one at the top and the other near the bottom (see fig.1). The oilwater mixture flows tangentially from the inlet into the cylindrical cyclone, and the denser component (water) tend to be accumulated near the wall due to a strong swirl and spiral down to the bottom (underflow), while a secondary vortex appears in the core region and exits from the top outlet (overflow).

Despite its simplicity in geometry, however, the multiphase flow behaviors in LLCC are actually very complex. In the past decades most of studies have been focused on Liquid-Liquid Hydro cyclones ${ }^{[2-5]}$, and very few studies have been published on LLCC. Firstly, researchers were mainly concerned about the influence of operating parameters or geometry changes on the separation result through experiments, and empirical or semi-empirical models were proposed to design the geometry and predict the separating performance. With the great progress in computer technology and the development of turbulent model, computational fluid dynamics (CFD) has gained more popularity in recent years ${ }^{[6-9]}$. Under appropriate turbulent models and proper boundary conditions, numerical simulation based on CFD has proven to be valuable in predicting the flow characteristics in a hydrocyclone ${ }^{[10]}$. Due to its lowcost and short period, CFD is widely used in designing and optimizing the cyclone ${ }^{[11-13]}$. In this study CFD technique is applied to simulate the liquidliquid flow in cylindrical cyclone with the Euler-Euler approach and the Reynolds stress model. Through numerical simulation the multiphase flow field is achieved and other operation parameters are studied on the influence of the separation efficiency.

\section{NUMERICAL SIMULATION}

\subsection{Basic equations}

The governing partial differential equations are the mass conservation equation and the momentum conservation equation, as follows: 


$$
\frac{\partial \rho}{\partial t}+\nabla \cdot \rho u=0
$$

$\frac{\partial \rho \vec{u}}{\partial t}+\nabla \cdot(\rho \vec{u} \vec{u})=-\nabla p+\nabla \cdot\left[\mu\left(\nabla u-\frac{2}{3} \nabla \cdot \rho \vec{u}^{2}\right)\right]+\rho \vec{g}$

The variables $\rho, \mathrm{p}$ and $\mu$ represent density, pressure and molecular viscosity, respectively.

Turbulence model: It is important to choose an appropriate turbulence model. For the strong swirling flow in a cyclone, the $\kappa-\varepsilon$ model based on eddyviscosity approach fails to predict the flow behaviors well. Therefore, the Reynolds Stress Model (RSM) is selected to capture the anisotropic character of the turbulence in the cyclone:

$$
\begin{aligned}
& \frac{\partial}{\partial t}\left(\rho \overline{u_{i}^{\prime} u_{j}^{\prime}}\right)+\frac{\partial}{\partial x_{k}}\left(\rho u_{k}^{\prime} \overline{u_{i}^{\prime} u_{j}^{\prime}}\right)=P_{i j}+D_{T i j}+\phi_{i j}-\varepsilon_{i j}+F_{i j} \\
& P_{i j}=-\rho\left(\overline{u_{i}^{\prime} u_{k}^{\prime}} \frac{\partial u_{j}}{\partial x_{k}}+\overline{u_{j}^{\prime} u_{k}^{\prime}} \frac{\partial u_{i}}{\partial x_{k}}\right) \\
& \left.D_{T i j}=-\frac{\partial}{\partial x_{k}}\left[\rho \overline{u_{i}^{\prime} u_{j}^{\prime} u_{k}^{\prime}}+\overline{p\left(\delta_{k j} u_{i}^{\prime}+\delta_{i k} u_{j}^{\prime}\right.}\right)\right] \\
& \phi_{i j}=\left(\overline{\frac{\partial u_{i}^{\prime}}{\partial x_{j}}+\frac{\partial u_{j}^{\prime}}{\partial x_{i}}}\right) \\
& \varepsilon_{i j}=-2 \mu \frac{\partial u_{i}^{\prime}}{\partial x_{k}} \frac{\partial u_{j}^{\prime}}{\partial x_{k}} \\
& F_{i j}=-2 \rho \Omega_{k}\left(\overline{u_{j}^{\prime} u_{m}^{\prime}} \varepsilon_{i k m}+\overline{u_{i}^{\prime} u_{m}^{\prime}} \varepsilon_{j k m}\right)
\end{aligned}
$$

The $P_{i j}$ term represents the stress production, $D_{i j}$ is the turbulent diffusion term, $\phi_{i j}$ is the pressure-strain term, $\mathcal{E}_{i j}$ is the viscosity diffusion term, $F_{i j}$ is the rotation production term.

\subsection{Cylindrical cyclone geometry}

A schematic of the LLCC is given in Fig. 1. The vertical cylinder is $1590 \mathrm{~mm}$ height and $100 \mathrm{~mm}$ ID, with an equal diameter horizontal inlet. The inlet is attached to the LLCC $160 \mathrm{~mm}$ below its top. A nozzle is placed at the inlet, causing the flow to enter the LLCC tangentially. The inlet slot area is $20 \%$ of the full cross sectional area. The diameters of overflow and underflow tube are $30 \mathrm{~mm}$ and $60 \mathrm{~mm}$, respectively. The underflow tube is located tangentially to the LLCC $80 \mathrm{~mm}$ above the bottom. A mesh of 155,906 computational cells is generated in Gambit. The whole geometry is split into four blocks and each block is then meshed separately. The inlet and underflow blocks choose tetrahedral cells, while the other blocks choose hexahedral cells.

Water and oil are chosen to be the test fluids. The primary phase is water with a density of $998.0 \mathrm{~kg} / \mathrm{m}^{3}$ and a viscosity value of $0.001 \mathrm{~kg} / \mathrm{m} \cdot \mathrm{s}$, while the second phase oil's density and viscosity are $836 \mathrm{~kg} / \mathrm{m}^{3}$ and $0.031 \mathrm{~kg} / \mathrm{m} \cdot \mathrm{s}$, respectively. The commercial software "Fluent 6.3.26" is used, which is based on finite element method to discretize the differential equations describing the multiphase flow. Currently, there are two approaches to simulate the multiphase flows: the Euler-Lagrange approach and the EulerEuler approach. The Lagrangian discrete phase model in Fluent follows the Euler-Lagrange approach. The primary phase is treated as a continuum, while the other is dispersed in the flow field in the form of particles, bubbles or droplets. The fundamental assumption in the model is that the volume fraction of the dispersed second phase is so low that its effect on the primary phase can be negligible. But it is inappropriate for the modeling of liquid-liquid mixtures where the volume fraction of the second phase is enough high to fully consider the phasic interactions. Therefore, the Euler-Euler approach, in which the different phases are treated as interpenetrating continua, is adopted for the present study focuses on the volume fraction of second phase more than 10 percent. At the entrance a "velocityinlet" boundary condition is applied. Flow rate at the two outlets are specified. No-slip boundary condition is assumed at the internal wall. The SIMPLEC algorithm is used to couple the pressure and velocity, and the second-order upwind scheme is applied to interpolate the field variables on faces of the control volumes. The iterative procedure will not stop until the continuity residual to $1.0 \times 10^{-6}$.

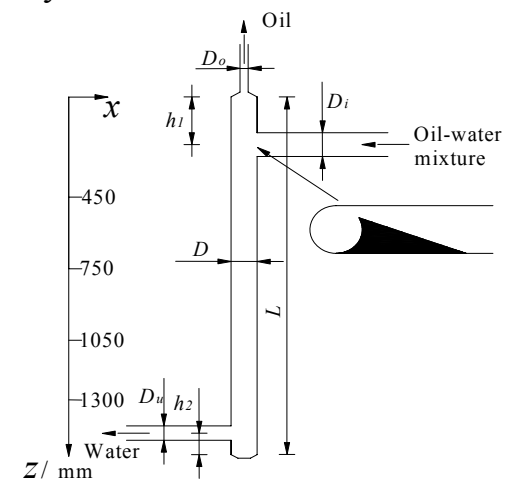

Fig. 1 Schematic of LLCC

\section{RESULTS AND ANALYSIS}

\subsection{Flow characteristics}

In the simulation, the inlet velocity is $2 \mathrm{~m} / \mathrm{s}$, and the volume fraction of second phase (oil) is 10 percent. The characteristics of the flow in the cyclone are obtained from the post-processing of the simulating data. In order to get a better understand of the flow in the cyclone, particle motions or particle trajectories are shown in Fig. 2. In this study, the second phase is 
assumed to be spherical particles (oil droplets) dispersed in water with a diameter of $0.5 \mathrm{~mm}$. As can be seen, the particles are tangentially injected into the cylindrical cyclone through the horizontal inlet tube and cause a strong swirling motion in the inner cyclone. Particles are divided into two parts, one part eject directly from the overflow tube while the other flow to the wall and down to the bottom in a helical path. In the downward flow, due to the effect of centrifugal force and pressure gradient some of the downward flow particles enter into the core, and flow up to the top outlet with the inner upward flow. From the number of trajectories, it is easy to achieve that most of the particles or mixture injected from the inlet flows out from the underflow tube.

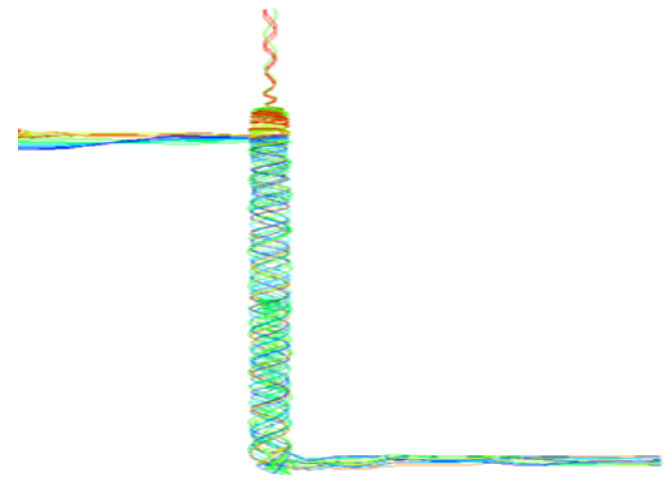

Fig. 2 Particles Trajectories

The pressure distribution in the cylindrical cyclone has an intimately relevant to energy transformation and diffusion. Fig. 3a shows the pressure contours of the axial cross section of cyclone. It can be seen that the pressure value near the cyclone wall is higher than the axial core zone, especially near the inlet the value is much higher. From the inlet to the bottom outlet, the pressure value near the wall decreases due to the wall friction energy lost and other energy diffusion. In the inner of the cyclone a low pressure zone is formed in the axial core. It is the low pressure zone that causes an inner upward flow. A pressure distribution line, which follows the center axis of the cyclone from the top to the bottom, is shown in Fig. 3b. A minus pressure zone is formed near the overflow, and with the distance to the bottom decreasing the pressure value increases and reaches a maximum near the bottom. There are intense fluctuations of the pressure value in the vicinity of the inlet and underflow tube due to the turbulent flow field.

To achieve the flow field of a cyclone is helpful to understand the separation mechanism. The flow is usually described as a combination of two parts: an outer helical downward flow and an inner helical upward flow. The velocity components, tangential, axial and radial flow velocity, are defined at the base of the geometry of cyclone ${ }^{[14]}$.

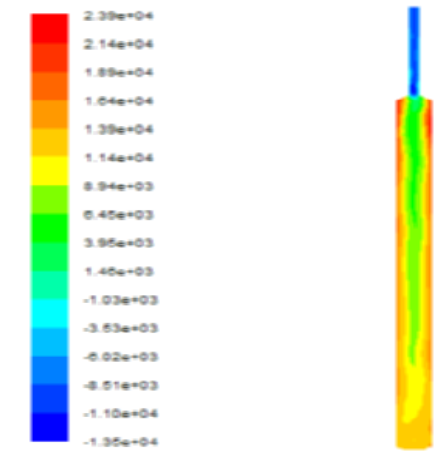

(a) Pressure contours

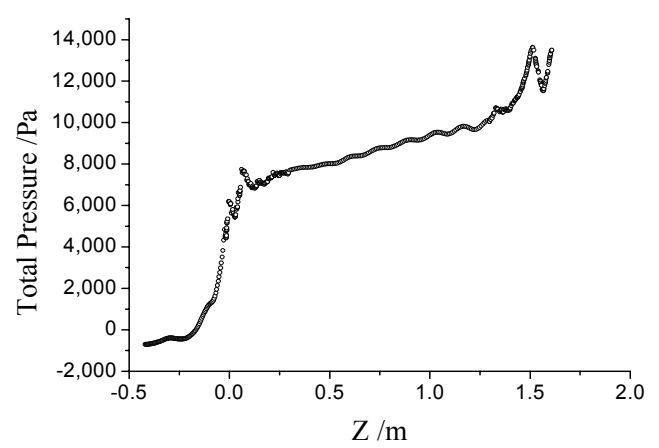

(b) Pressure distribution along with the axial center line Fig. 3 Pressure distribution of the axial cross section

Tangential velocity is considered to be the most important component inside a cyclone, as it decides the value of centrifugal acceleration and force. Fig. 4 shows the tangential velocity distribution along with the radii at a height of the cyclone. As can be seen, the tangential velocity is approximately axial symmetry distributed. It can be attributed to a certain distance far from the inlet part and the inlet asymmetry effect plays little role on the velocity distribution. Because of the stationary wall and the effect of boundary layer, the tangential velocity is zero near the wall and increases to a maximum along with the radii, and then decreases to the minimum close to the center. In this cylindrical cyclone, the tangential velocity does not present a forced vortex and a free vortex apparently, and this phenomenon has a difference to the tangential velocity distribution of hydrocyclone reported by several researchers ${ }^{[15,16]}$.

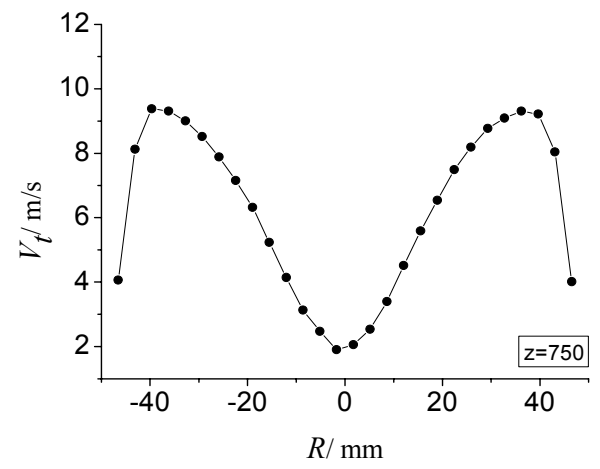

Fig. 4 Tangential velocity distribution 
Axial velocity distribution is shown in Fig. 5. From the cyclone wall to the center the axial velocity increases from zero to a maximum value firstly, and decreases to zero again at the place of about half radii. Then the value turns to be minus and the absolute value increases until at the center reaches a maximum. All the zero axial velocity form a zero axial velocity envelope surface. There are counter-current axial flows in the both sides of the envelope surface, including an outer downward flow and an inner upward flow. During oil-water separation in cyclone oil core is formed in the upward flow.

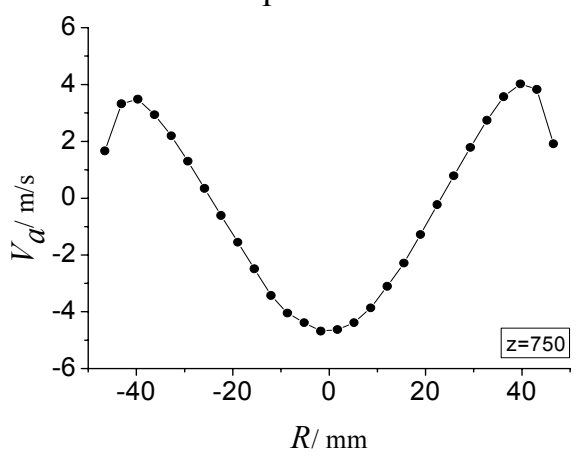

Fig. 5 Axial velocity distribution

Fig. 6 shows the radial velocity distribution. It can be seen that the radial velocity value increases along with the radii, but near the center the value decreases to zero rapidly. This is because oil droplets are draught to the inner part and form oil core flowing upward with the inner helical flow. The radial velocity distribution presents point symmetry about the center. Comparing with tangential and axial velocity, the value of radial velocity is much smaller about one order. For this reason studies about measuring the radial velocity by experiment method are fewer. A further explanation for this could be attributed to the smaller value and difficult to measure.

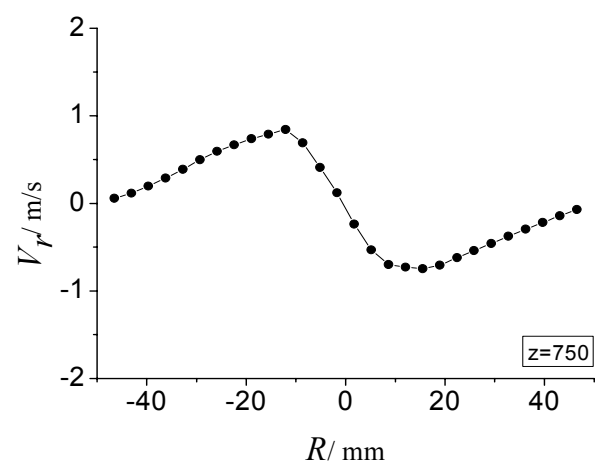

Fig. 6 Radial velocity distribution

\subsection{Phase distribution}

Oil-water mixture is injected tangentially into the cyclone and a strong swirling motion is formed. Due to the difference of density, oil and water are separated by the centrifugal force. Fig. 7 a shows the volume fraction contours of oil phase in different cross sections. Most oil phase flow into the inner to form an oil core. In the same cross section, oil volume fraction is high at the center and much lower near the wall. The oil core is helical and accumulated more intensively near the inlet part. Along with the height of cyclone the oil core turns to be slender and disappears at the upper of the underflow. Fig. $7 \mathrm{~b}$ is an oil core working sketch. The oil core through numerical solution is similar and consistent with the observation in lab.

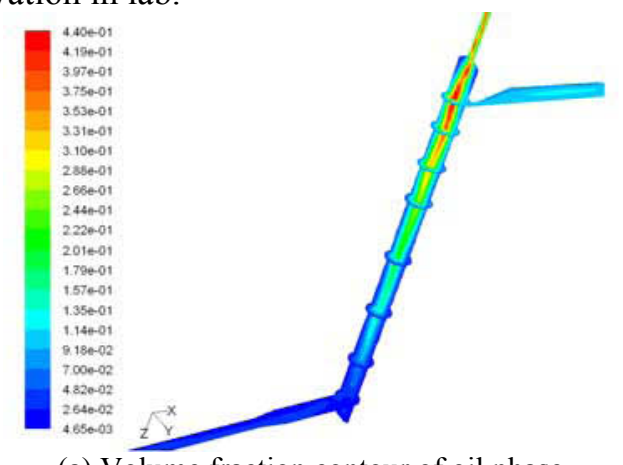

(a) Volume fraction contour of oil phase

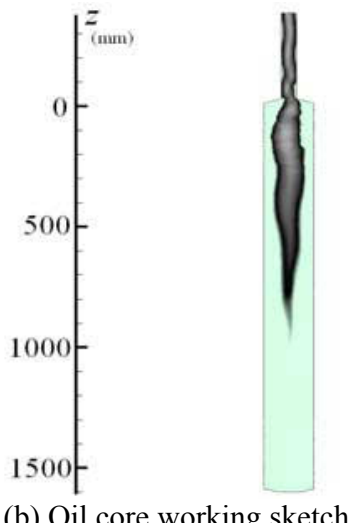

Fig. 7 Oil-water contours in different cross sections

\subsection{Separation performance}

Despite the simplicity in geometry, there are lots of factors to affect the separation performance, including operation parameters, geometry parameters and fluid characteristic parameters. In the following operation parameters, flow split-ratio and non-dimensional separation acceleration, are discussed.

Flow split-ratio $(f s)$ is defined as the ratio between the overflow liquid flow rate and the inlet liquid flow rate, as given by

$$
f_{s}=\frac{Q_{o}}{Q_{i}}
$$

Flow split-ration, which influences directly the capacity and separation efficiency of cyclone, is one of the important operation parameters. In this study the separation efficiency is defined as:

$\eta=1-\frac{C_{o}}{C_{i}}$ 
Where $C_{i}$ is the oil volume fraction of inlet flow, and $C_{o}$ is the oil volume fraction of overflow. Fig. 8 shows the effect of the split-ration on separation efficiency. The flow split-ratio ranges from 0.2 to 0.5 . The curve indicates that at smaller split-ratios oilwater in cyclone cannot be separated efficiently. Increasing the split-ratio can dramatically improve the separation. While the split-ration reaches a certain value, the separation efficiency increases slowly with further increasing split-ratio. This shows that there is an appropriate split-ratio for cyclone. According to the efficient curve the optimal split-ratio is 0.4 for the cylindrical cyclone's geometry.

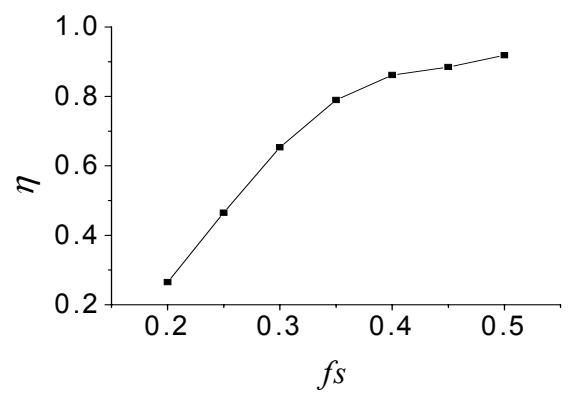

Fig. 8 Relationship between split-ratio and separation efficiency

Non-dimensional separation acceleration, also called $\mathrm{G}$-force is defined as the ratio between centrifugal acceleration and gravitational acceleration, as follows:

$G_{f}=\frac{a_{c}}{g}$

Where $\mathrm{g}$ is the gravitational acceleration, and $a_{c}$ is the centrifugal acceleration, and is calculated as:

$a_{c}=\frac{V_{t}^{2}}{R}$

The parameter $V_{t}$ is the tangential velocity, and $\mathrm{R}$ is the radii of the cyclone. G-force is often used to measure the intensity of swirling motion in cyclone. Fig. 9 shows the effect of G-force on separation efficiency. Four different G-force values are considered in this study, listing as 51, 204, 459, 816. The oil-water separation efficiency of the cyclone increases with increasing the G-force. There is a rapid augment of separation efficiency when the G-force value increases from 51 to 204, while from 459 to 816 the separation efficiency increases much slowly. This is because the mixture is oil-water two phase flows. When oil-water mixture is injected at extremely high velocity, there will be emulsification phenomena due to the intensive shear effect and oil-water separation is deteriorated. During numerical simulation emulsification and droplet break-up are out of consideration. A further study should include the effect of emulsification and droplet break-up to improve simulating separation.

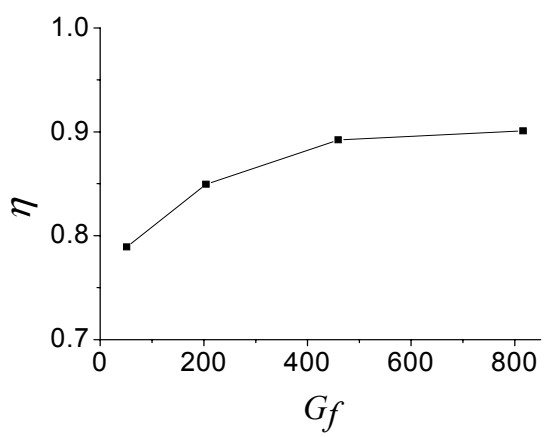

Fig. 9 Relationship between G-force and separation efficiency

\subsection{Model validation}

To validate the methodology above, a geometry described in detail in literature C.O. Vazquez (2004) ${ }^{[17]}$ was adopted to simulate the oil-water separation in cylindrical cyclone. Fig. 10 presents the comparison of images obtained from numerical simulations and experiments. The superficial velocities of oil and water are $0.32 \mathrm{~m} / \mathrm{s}$ and $1.0 \mathrm{~m} / \mathrm{s}$, respectively. In the first case, the flow split-ratio is 0.5 , while 0.6 in the second case. As can be seen, there is good agreement with respect to the phase distribution between the results from numerical simulations and experiments.
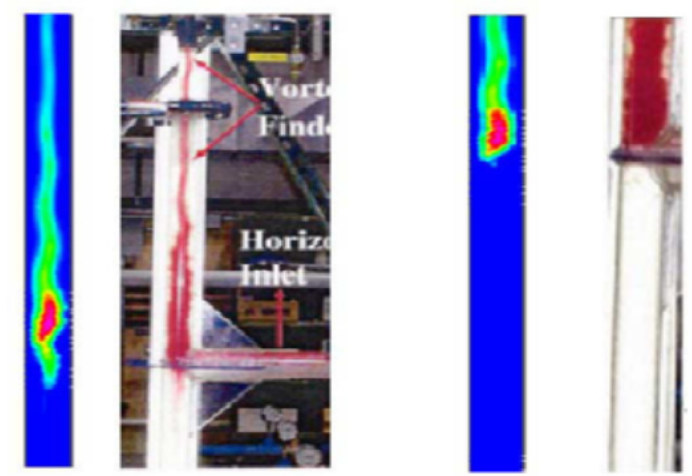

Fig. 10 Phase distribution comparison from numerical solution and experiments

\section{CONCLUSIONS}

A kind of liquid-liquid cylindrical cyclone has been proposed and further studied through numerical simulations integrating the Euler-Euler approach and the RSM turbulence model. Through investigating the flow field in cyclone a better understand of oil-water separation mechanism is reached. The following conclusions can be drawn.

(1) The separation efficiency increases with increasing the flow split-ratio. There is an appropriate split-ratio for a cyclone with certain geometry.

(2) The proper increase of G-force can improve oilwater separation in a cyclone. However, a extreme high G-force may deteriorate the separation due to 
emulsification.

(3) The effect of emulsification and droplet breakup should be considered in numerical simulation to improve simulating separation.

\section{ACKNOWLEDGEMENT}

The authors are grateful for the financial support provided by the Key Project of the "Eleventh FiveYear Plan" of the Chinese Academy of Sciences (Grant No. KJCX2-YW-L02).

\section{REFERENCES}

[1] C Oropeza-Vazquez, E Afanador, L Gomez, et al. Oil-water separation in a novel liquid-liquid cylindrical cyclone (LLCC) compact separator-experiments and modeling [J]. Journal of Fluids Engineering, 2004, 126, 553-564.

[2] $\mathrm{M} \mathrm{T}$ Thew. Hydrocyclone redesign for liquid-liquid separation[J]. The Chemical Engineer, 1986, 17-19,21,23.

[3] L R Castilho, R A Medronho. A simple procedure for design and performance prediction of Bradley and Rietema hydrocyclones[J]. Minerals Engineering, 2000, 13(2): 183-191.

[4] Liang-Yin Chu, Wen-Mei Chen, Xiao-Zhong Lee. Effect of structural modification on hydrocyclone performance[J]. Separation and Purification Technology, 2000,21: 71-86.

[5] A Belaidi, M T Thew. The effect of oil and gas content on the controllability and separation in a de-oiling hydrocyclone[J]. Trans I Chem E, 2003, 81: 305-314.

[6] J C Cullivan, R A Williams, C R Cross. Understanding the hydrocyclone separator through computational fluid dynamics [J]. Institution of Chemical Engineers, 2003, 81: 455-466

[7] S Schuetz, G Mayer, M Bierdel, et al. Investigations on the flow and separation behavior of hydrocyclones using computational fluid dynamics [J]. Int. J. Miner Process,
2004, 73: 229-237.

[8] Miguel A Reyes, Jorge E Pacheco, Juan C Marín, et al. Numerical simulation and experiments of the multiphase flow in a liquid-liquid cylindrical cyclone separator [A]. Proceedings of European Fluids Engineering Summer Meeting [C], Miami, Florida, 2006: 1-7.

[9] M Brennan. CFD simulations of hydrocyclones with an air core comparison between large eddy simulations and a second moment closure [J]. Chemical Engineering Research and Design, 2006, 84: 495-505

[10] A F Nowakowski, J C Cullivan, R A Williams, et al. Application of CFD to modeling of the flow in hydrocyclones [J]. Minerals Engineering, 2004, 17: 661669.

[11] Charles L Karr, Donald A Stanley, Brian McWhorter. Optimization of hydrocyclone operation using a genofuzzy algorithm [J]. Comput. Methods Appl. Mech. Engrg. 2000, 186: 517-530.

[12] T J Olson, R Van Ommen. Optimizing hydrocyclone design using advanced CFD model [J]. Minerals Engineering, 2004, 17: 713-720.

[13] Jose A Delgadillo, Raj K Rajamani. Exploration of hydrocyclone designs using computational fluid dynamics [J]. Int. J. Miner Process, 2007, 84: 252-261.

[14] Jonas Bergström, Hannes Vomhoff. Experimental hydrocyclone flow field studies [J]. Separation and Purification Technology, 2007, 53(1): 8-20.

[15] D F Kelsall. A study of the motion of solid particles in a hydraulic cyclone[J]. Trans. Inst. Chem. Eng, 1952, 30: 87-108.

[16] B Dabir, C A Petty. Measurement of mean velocity profiles in a hydrocyclone using laser Doppler anemometry [J]. Chem. Eng. 1986, 48: 377-388.

[17] Carlos Oropeza-Vazquez. Multiphase flow separation in liquid-liquid cylindrical cyclone and gas-liquid-liquid cylindrical cyclone compact separators [C]. Chem. Eng. 1986, 48: 377-388. Ph. D. Thesis, Tulsa, The University of Tulsa, 2001. 